\title{
Japanische Popkultur: \\ Raum für Vielfalt und Grenzüberschreitungen
}

\section{Michiko Mae und Elisabeth Scherer}

Die japanische Populärkultur hat in den letzten Jahren in den USA und Europa einen Siegeszug angetreten, der frühere Blütezeiten der Japan-Begeisterung wie z. B. den Japonismus vor hundert Jahren noch übertrifft. Ihre Produkte sind längst Teil der westlichen Mainstream-Kultur und werden von vielen Fans als eine Alternative zur bisher dominanten amerikanischen Popkultur gesehen. Auch die japanische Regierung unterstützt inzwischen offiziell den Export von Anime und Manga, die sie als wichtige Repräsentationen der modernen japanischen Kultur betrachtet.

Während junge deutsche Fernsehzuschauer in den 1970er und 1980er Jahren wahrscheinlich keine Ahnung davon hatten, dass ihre Zeichentrickheldinnen Biene Maja und Heidi in japanischen Studios das Licht der Welt erblickt hatten, gehören die Autorinnen und Autoren dieses Bandes einer Generation an, die japanische Populärkultur bereits seit ihrem Jugendalter bewusst konsumiert. Das Vorurteil, Videospiele und Anime seien Schund, ist längst überholt, und inzwischen sind die populärkulturellen Medien auch ein ernst zu nehmendes Objekt für wissenschaftliche Analysen geworden. Das hängt auch mit der atemberaubenden Entwicklung der technischen Möglichkeiten zusammen, liegt aber vor allem an der Qualität der komplexen und facettenreichen Narrationen in den genannten Genres. Sie greifen in ihren unterschiedlichen Ausdrucksmöglichkeiten aktuelle gesellschaftliche Themen und Probleme auf und weisen sogar auf Lösungsmöglichkeiten hin, auch indem sie neue Identifikationsfiguren anbieten.

Diese neue Qualität der japanischen Popkultur wurde schnell von den jungen Rezipient/innen erkannt. Auch westliche Fans können sich mit japanischen Comic-Figuren identifizieren, wie man auf Conventions oder in Internet-Foren beobachten kann. Nicht wenige Fans übersetzen japanische Mangas (oder kre- 
ieren sogar ihre eigenen) und sind sich dabei gerade der spezifisch japanischen Elemente dieses Genres bewusst.

Die Themen der neuen populärkulturellen Medienprodukte haben ein schier unüberschaubar breites und vielfältiges Spektrum. Die Beiträge im vorliegenden Band befassen sich mit solchen Werken, die im Zusammenhang mit dem am Institut für Modernes Japan angesiedelten Projekt „Neue Differenzen und Diversität in der japanischen Gesellschaft" interessant sind. Der Umgang mit Differenz und Diversität ist gerade in Bezug auf die japanische Gesellschaft ein wichtiges Thema, weil Japaner lange Zeit hartnäckig behauptet haben, ihr Land zeichne sich durch eine besondere Homogenität aus. Die japanische Kultur und Gesellschaft waren aber in keiner historischen Phase wirklich homogen; ganz im Gegenteil lag gerade in ihrer Heterogenität und Vielfalt das, was sie in besonderer Weise prägte. Darüber hinaus nehmen im Zeitalter der Globalisierung in Japan Heterogenität und Vielfalt sehr stark zu.

Dieser Wandlungsprozess zeigt sich auch in den vorliegenden Analysen. Es geht darin um unterschiedliche Umgangsweisen mit kulturellen, gesellschaftlichen sowie Gender-bezogenen Differenzen und mit der wachsenden soziokulturellen Vielfalt. Die zunehmende Diversität, die sich durch das Zusammenleben mit kulturell-ethnischen Minderheiten ergibt, spiegelt sich in den populärkulturellen Produkten ebenso wider wie die Auflösung der japanischen Mittelschicht, die lange als Fundament der vermeintlich homogenen Gesellschaft galt. Auch die Frage der Geschlechteridentität ist bereits seit langem ein Thema, zum Beispiel in Comics für junge Mädchen, den sogenannten shōjō manga. Mit der Diskussion um das 1999 in Kraft getretene Partizipationsgesetz, dessen Ziele die völlig gleichen Beteiligungs- und Gestaltungsmöglichkeiten in der Gesellschaft unabhängig vom Geschlecht sind, fand das Thema Männer- und Frauenrollen und identitäten auch Eingang in Mainstream-Medien wie Fernsehserien. Und auch die mediale Verarbeitung von Tabuthemen wie zum Beispiel häusliche Gewalt trägt in Japan dazu bei, das Bewusstsein der Zuschauer zu schärfen und Handlungsbarrieren abzubauen.

Im Folgenden sollen die einzelnen Beiträge dieses Bandes und die darin aufgegriffenen gesellschaftlichen Tendenzen kurz vorgestellt werden. 
Ebenso wie der vorliegende Band trägt der Manga Shōjo kakumei Utena, den Melissa Sohlich analysiert, das Wort „Revolution“ (kakumei) im Titel. Sohlich zeigt auf, worin in dem Werk, das eine hochkomplexe Handlung aufweist, das Revolutionäre besteht. Dabei werden vor allem durch die Hauptfigur Utena Stereotype, die aus der Märchenwelt bekannt sind, aufgebrochen und die Erschaffung einer neuen, freien Identität jenseits von vorgegebenen Rollenbildern möglich gemacht.

Katharina Hülsmann untersucht das Videospiel Silent Hill 4: The Room und stößt dabei auf Gestaltungselemente, die auf eine Auseinandersetzung mit dem hikikomori-Phänomen hinweisen. Hikikomori sind junge Menschen, die sich aus der Gesellschaft zurückziehen und deren Alltag durch einen sehr hohen Medienkonsum geprägt ist. Videospiele und virtuelle Welten sind die vermeintlichen Schutzräume für solche sozial isolierten Jugendlichen. Dadurch, dass das Design von Silent Hill 4 den Weg aus einem solchen Raum simuliert, erhält das Spiel selbstreflexive Züge.

Um unheimliche Wesen, sogenannte yōkai, geht es in dem Beitrag von Timo Thelen. Während diese Flusskobolde, Berggeister und Langhalsgespenster in der Edo-Zeit (1603-1868) noch sehr gefürchtete Gestalten waren, durchlaufen sie heute zunehmend eine "Verniedlichung" und werden zu Symbolen von unberührter Natur, „Japanizität" und Heimat. Thelen zeigt dies anhand des Anime Miyori no Mori auf, in dem ein Mädchen gemeinsam mit den yōkai gegen die rücksichtslose Rodung eines Waldes ankämpft.

Einem bisher wenig untersuchten Gebiet widmet sich Marvin Udzik in seinem Beitrag zu Repräsentationen von Geschlechterrollen im japanischen Videospiel. Bis vor wenigen Jahren beschränkte sich die Funktion weiblicher Charaktere in Computerspielen häufig darauf, von einem männlichen Helden aus den Klauen einer bösen Macht befreit zu werden. Ein klassisches Beispiel für eine solche "Jungfrau in Gefahr“ ist Prinzessin Peach in den Spielen der Super-MarioSerie. Wie Udzik aufzeigt, tauchen seit der Jahrtausendwende aber auch starke und selbständige weibliche Charaktere auf, die in ihrer Gender-Rolle ambivalent auftreten und Aspekte neuer Geschlechterbilder in sich tragen. 
Die (Um-)Konstruktion von Männlichkeit vor dem Hintergrund der japanischen Polarisierungsgesellschaft (kakusa shakai) ist Thema des Aufsatzes von Bastian Nonnenberg. Am Fallbeispiel der Fernsehserie Serebu to binbō tarō („Die Prominente und der mittellose Tarō“, Fuji TV, 2008) zeigt Nonnenberg verschiedene Kategorien von Männlichkeit(en) auf, denen die Serienfiguren zugeordnet werden können. Im Ergebnis erweist sich Serebu to binbō Tarō im Gegensatz zu den meisten anderen in diesem Band behandelten Werken als stark reaktionär. Zwar wird als Ausgangssituation ein Paar beschrieben, bei dem der Mann der Frau wirtschaftlich stark unterlegen ist. Eine Lösung der sich aus dieser Lage ergebenden Konflikte ist aber nur durch eine Wiederherstellung konservativer Muster möglich.

Fauve Görlach befasst sich damit, wie in der Fernsehserie Last Friends (Fuji TV, 2008) anhand der Figur Michiru die Situation eines Opfers von häuslicher Gewalt geschildert wird. Sie zeigt auf, dass die Entwicklung der Gewaltspirale und das Verhalten von Täter und Opfer sehr realitätsnah aufgezeigt werden, wodurch die Serie einen aufklärenden Charakter erhält und möglicherweise sogar dazu beitragen kann, das Bewusstsein der japanischen Gesellschaft für diese Problematik zu schärfen. Weitere wichtige Themen der Serie sind Geschlechteridentität bzw. Homosexualität und Freundschaft. Das innovative Konzept der Serie wird vor allem am Ende deutlich, wenn das Trio Michiru, Takeru und Ruka gemeinsam Michirus Kind aufzieht und damit ein alternatives Familienmodell verkörpert, das in Japan als revolutionär bezeichnet werden kann.

Den Multikulturalismus in dem Film Swallowtail Butterfly (Iwai Shunji, 1996), der von dem Leben asiatischer Einwanderer in Japan handelt, untersucht Alexander Fegler. Schonungslos wird in Iwais Werk aufgezeigt, welche Folgen es zeitigen kann, wenn ethnische Minderheiten abgesondert von der gesellschaftlichen Mitte, hier repräsentiert durch die japanische „Downtown“, in einen Raum des „Fremden“ gedrängt werden. Fegler sieht in Swallowtail Butterfly ein Plädoyer für interkulturelle Toleranz und die Anerkennung von Individualität.

Afroamerikanische Figuren stehen im Mittelpunkt des Romans Bedtime Eyes (Yamada Amy, 1985) und des Films Blues Harp (Miike Takashi, 1998). Adam Jambor stellt in seinem Beitrag zu diesen Werken fest, dass Yamada Amy die 
Eigenheiten ihres afroamerikanischen männlichen Protagonisten als besonders exotisch und anziehend darstellt, während Miike Takashi diese ethnischen und kulturellen Differenzen aufhebt und eine Art Postethnizität erschafft.

Um die Probleme koreanischer Einwanderer dreht sich der Film Blood \& Bones (Sai Yōichi, 2004), mit dem sich Daniel Steinhäuser in seinem Beitrag auseinandersetzt. Mit dem extremen Einsatz von Gewalt sichert sich der Protagonist Shunpei seine patriarchalischen Herrschaftsansprüche innerhalb der Familie und der koreanischen Gemeinde. Diese im Film thematisierte Hypermaskulinität liegt nach Steinhäusers Ansicht darin begründet, dass sich Shunpei durch seinen speziellen Status als Japankoreaner gesellschaftlich ausgegrenzt und in seiner Männlichkeit marginalisiert fühlt.

Alle diese Beiträge zeigen, dass die japanische Populärkultur eine große Vielfalt ermöglicht und sich immer häufiger über bestehende Stereotype hinwegsetzt. In Manga, Fernsehserien und Filmen können Dinge zur Sprache kommen, die in der gesellschaftlichen Realität oft noch mit Tabus besetzt sind, wie zum Beispiel der Umgang mit Homosexualität oder häuslicher Gewalt. Dabei offenbart sich ein subversives Potential, das nicht im Bereich der Fiktion verbleiben muss, sondern auch tatsächliche „revolutionäre“ Veränderungen im Denken und Fühlen junger Japaner/innen bewirken kann. 
